\title{
A Simple Route to Asymmetric Composite Particles via Co-assembly of Block Copolymer and Inorganic Nanoparticles in Solution
}

\author{
Chun-Sheng $\mathrm{LI}^{\mathrm{a}, \star}$, Wen-Ting LI, Jun LIU, Xue LI
}

School of Chemistry and Chemical Engineering, University of Jinan, No. 336 West Road of Nan Xinzhuang, Jinan 250022, People's Republic of China

achm_lics@ujn.edu.cn

${ }^{*}$ Corresponding author

Keywords: Block Copolymers, Asymmetric Particles, Microphase Separation, $\mathrm{Fe}_{3} \mathrm{O}_{4}$.

\begin{abstract}
A novel approach to organic-inorganic asymmetric particles through the co-assembly of block copolymers (BCs) and magnetic $\mathrm{Fe}_{3} \mathrm{O}_{4}$ nanoparticles (NPs) in solution is demonstrated. When 11-mercaptoundecanoic acid (MUA) modified $\mathrm{Fe}_{3} \mathrm{O}_{4} \mathrm{NPs}$ and an amphiphilic block copolymer polystyrene-b-poly (2-vinylpyridine) (PS-b-P2VP) were mixed in THF, asymmetric composite particles formed due to the hydrogen bonding interactions between $-\mathrm{SH}$ groups of the MUA molecules coated on $\mathrm{Fe}_{3} \mathrm{O}_{4}$ NPs and P2VP blocks. The asymmetric particles obtained by this facile approach may have potential applications in biomedical areas.
\end{abstract}

\section{Introduction}

Recently, asymmetric multicomponent nanoparticles (AMNPs) have attracted much attention due to their potential applications. Great efforts have been made in the preparation of inorganic-inorganic and organic-organic AMNPs. In contrast, the synthesis of polymer/inorganic hybrid AMNPs still remains a great challenge ${ }^{[1]}$ Several studies have already been published on polymer/inorganic hybrid AMNPs. Most of the methods are template-assisted, template modification, dispersion polymerization or miniemulsion polymerization. For example, Polystyrene (PS)- $\mathrm{SiO}_{2}$ hybrid asymmetric dimers could be prepared via a template-directed self-assembly technique. $^{[2]}$ Poly(2-vinylpyridine)- $b$-poly(ethylene oxide)/Au Janus micelles based on the protonation/deprotonation process of amphiphilic diblock copolymers in THF were prepared. ${ }^{[3]}$ Very recently, a novel approach to organic-inorganic Janus-like particles based on the alkalization process of PS- $b-\mathrm{P} 2 \mathrm{VP} / \mathrm{FeCl}_{3}$ micelles in toluene has also been presented. ${ }^{[4]}$

To date, asymmetric particles embedding superparamagnetic materials have attracted great interest because of their potential applications. ${ }^{[5]}$ However, it still remains a challenge to prepare asymmetric multicomponent magnetic inorganic/polymer composite NPs.

In this study we describe a simple strategy for the synthesis of asymmetric BC/magnetic NPs composite particles through the co-assembly of a BC and magnetic NPs in solution. MUA-modified $\mathrm{Fe}_{3} \mathrm{O}_{4}$ NPs and PS- $b$-P2VP were mixed in THF. The hydrogen bonding interactions between the $-\mathrm{SH}$ groups of the MUA molecules coated on magnetic NPs and P2VP changed the solubility of $\mathrm{P} 2 \mathrm{VP}$ which then self-assembled into asymmetric composite particles.

\section{Experimental Part}

\section{Materials}

PS- $b$-P2VP was purchased from Polymer Source. The average molecular weights of the PS block and P2VP block were 8200 and 8300 , respectively. $\mathrm{Fe}(\mathrm{CO})_{5}$, oleic acid, oleylamine, MUA, isopropanol, ethanol, hexane, toluene and THF were of analytical grade and used as-received.

\section{Sample Preparation}

Synthesis of $\mathrm{Fe}_{3} \mathrm{O}_{4}$ NPs: $\mathrm{Fe}_{3} \mathrm{O}_{4}$ NPs were synthesized according to S. Sun et al. ${ }^{[6]}$ 
Modification of $\mathrm{Fe}_{3} \mathrm{O}_{4}$ NPs: Excess MUA solution in THF was added into $\mathrm{Fe}_{3} \mathrm{O}_{4}$ NPs suspension, and then the modified NPs were washed several times with ethanol by centrifugation. Finally the resulting NPs (noted as $\mathrm{M}-\mathrm{Fe}_{3} \mathrm{O}_{4}$ ) were dispersed in ethanol.

Preparation of $\mathrm{BCP} / \mathrm{Fe}_{3} \mathrm{O}_{4}$ composite particles: The magnetic NPs were mixed with the $0.1 \mathrm{wt} \%$ PS- $b$-P2VP solution in THF, stirred for $48 \mathrm{~h}$ and then equilibrated for $24 \mathrm{~h}$.

\section{Characterization}

Transmission electron microscopy (TEM), high resolution TEM (HRTEM) and energy dispersive spectroscopy (EDS) were performed on an H-800 or a JEM-2100 electron microscopy. Fourier transform infrared (FTIR) spectra measurements were made on a Spectrum One FTIR spectrometer using $\mathrm{KBr}$ pellets. Dynamic light scattering (DLS) measurements were measured with a Zetasizer 3000HS, Malvern Instruments, Ltd., UK.

\section{Results and Discussion}

\section{Synthesis and Modification of $\mathrm{Fe}_{3} \mathrm{O}_{4}$ Nanoparticles}

Fig.1 is a TEM image of the $\mathrm{Fe}_{3} \mathrm{O}_{4}(\sim 10 \mathrm{~nm})$ synthesized with application of oleic acid and oleylamine as stabilizing agents. To increase the interaction between PS- $b$-P2VP BCPs and $\mathrm{Fe}_{3} \mathrm{O}_{4}$ NPs, the pre-synthesized $\mathrm{Fe}_{3} \mathrm{O}_{4}$ NPs were modified with MUA. The FTIR spectra (Fig. 2A) showed that the peak at ca. $3438 \mathrm{~cm}^{-1}$, which is ascribed to the $\mathrm{O}-\mathrm{H}$ or S-H stretch of the MUA ligands, becomes stronger after modification. The peaks between 3000 and $2800 \mathrm{~cm}^{-1}$ are attributable to the symmetric and antisymmetric methylene stretch. ${ }^{[7]}$ The presence of the large band in the range of 500 to $750 \mathrm{~cm}^{-1}$ illustrates the formation of a covalent gold-sulfur bond. ${ }^{[8]}$ The ESD result (Fig.2B) also shows that the particle contained $\mathrm{Fe}, \mathrm{O}, \mathrm{C}$ and $\mathrm{S}$ elements.

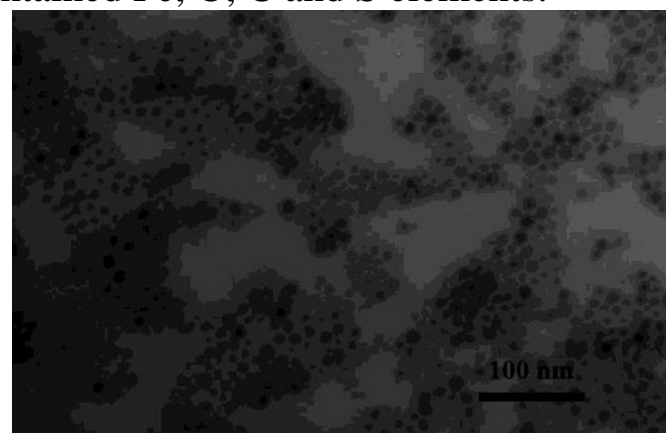

Fig.1 TEM Image of $\mathrm{Fe}_{3} \mathrm{O}_{4}$ NPs with Oleic Acid as Stabilizing Agent
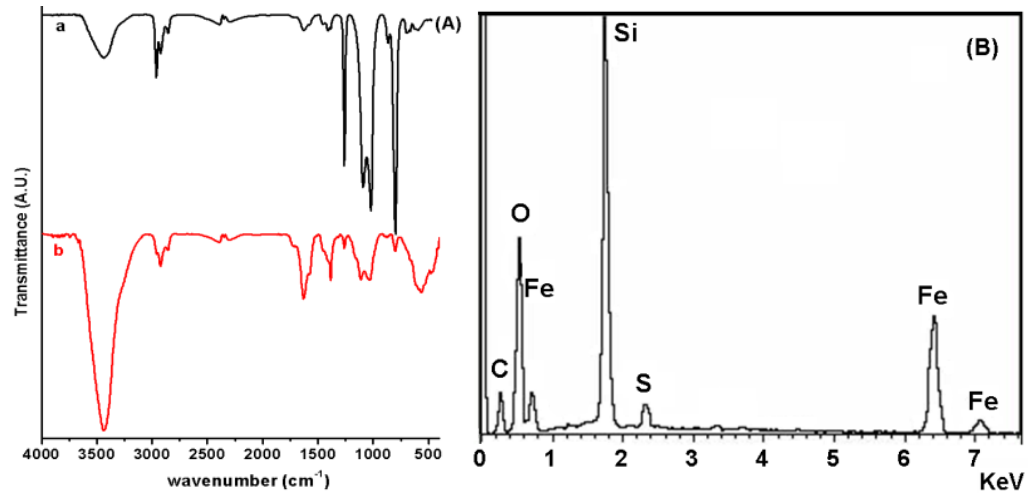

Fig.2 (A) FTIR Spectra of $\mathrm{Fe}_{3} \mathrm{O}_{4}$ NPs before (a) and after (b) Modification with MUA Molecules (B) EDS of $\mathrm{Fe}_{3} \mathrm{O}_{4}$ NPs after Modification with MUA Molecules

\section{Preparation of Asymmetric PS- $b$-P2VP/M-Fe $\mathrm{O}_{4}$ Composite Particles}

THF is a neutral solvent for PS and P2VP blocks. The DLS result revealed that most of the 
PS- $b$-P2VP molecules were present as unimers of about $4.8 \mathrm{~nm}$ diameter. If the pre-synthesized $\mathrm{Fe}_{3} \mathrm{O}_{4}$ NPs were mixed with PS- $b$-P2VP in THF, a precipitate was observed. One can see in Fig. 3a that individual $\mathrm{Fe}_{3} \mathrm{O}_{4} \mathrm{NPs}$ and random $\mathrm{Fe}_{3} \mathrm{O}_{4}$ NPs aggregates around the polymers were formed. The small circle in the large particle may be formed during solvent evaporation process. After removal of the precipitate, DLS measurement was performed. The appearance of two peaks (at $\sim 36$ $\mathrm{nm}$ and $190 \mathrm{~nm}$ ) (Fig. 3b) revealed that some aggregates formed in solution. Due to the weak interaction between the organic shells (the carbon chains of oleic acid) of $\mathrm{Fe}_{3} \mathrm{O}_{4}$ NPs and the P2VP or PS blocks, the $\mathrm{Fe}_{3} \mathrm{O}_{4}$ NPs tended to aggregate and precipitate out in THF giving rise to the 190 nm peak shown in Fig. $3 b$.

After the $\mathrm{Fe}_{3} \mathrm{O}_{4} \mathrm{NPs}$ were modified by using MUA molecules, PS- $b$-P2VP/M-Fe $\mathrm{O}_{4}$ mixtures in THF with varied amounts of $\mathrm{M}-\mathrm{Fe}_{3} \mathrm{O}_{4}$ were prepared. When the content of $\mathrm{M}-\mathrm{Fe}_{3} \mathrm{O}_{4}$ was 1.25 wt $\%$, the mixture solution was clear. One can see from Fig. 4a that $\mathrm{M}-\mathrm{Fe}_{3} \mathrm{O}_{4} \mathrm{NPs}$ assembled on one or two sides of PS- $b$-P2VP spheres rather than forming micellar or core-shell structures, indicating the formation of the biphasic structure. The average diameter measured by DLS is in the range from $100 \mathrm{~nm}$ to $820 \mathrm{~nm}$ (Fig. 6a, below). After careful observation of the sample with HRTEM, different shapes of the M-Fe ${ }_{3} \mathrm{O}_{4}$ NPs aggregates on the surface of PS- $b$-P2VP spheres were found (Fig. 4b-e). A higher magnification image (Fig. 4f), taken from Fig. 4e, reveals that $\mathrm{M}-\mathrm{Fe}_{3} \mathrm{O}_{4} \mathrm{NPs}$ (dark dots) assembled on the surface of PS- $b$-P2VP spheres.
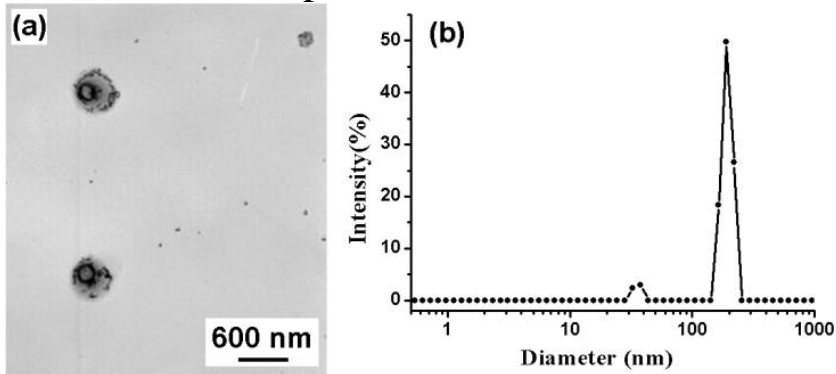

Fig.3 (a) TEM Images of the PS- $b$-P2 VP/Fe ${ }_{3} \mathrm{O}_{4}$ Aggregates Prepared from THF; the Contents of $\mathrm{Fe}_{3} \mathrm{O}_{4}$ NPs Relative to PS- $b$-P2VP are $1.25 \mathrm{wt} \%$ (b) DLS Size Distributions of the

$\mathrm{PS}-b-\mathrm{P} 2 \mathrm{VP} / \mathrm{Fe}_{3} \mathrm{O}_{4}$ Aggregates in THF

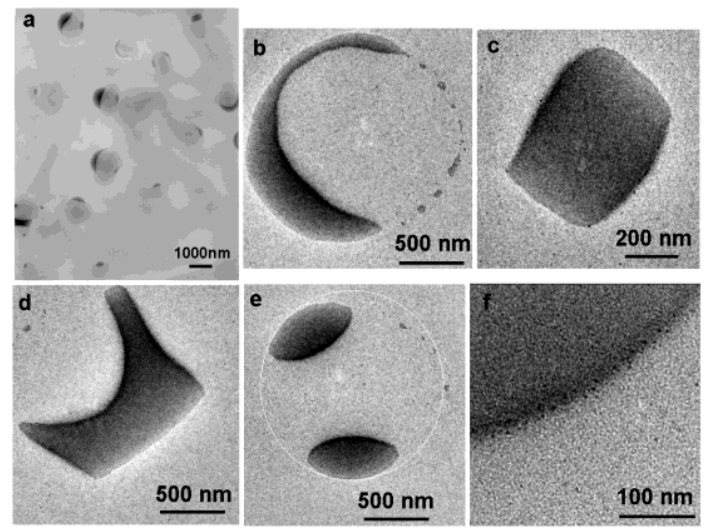

Fig.4 (a) TEM Image of the PS- $b$-P2VP/M-Fe $\mathrm{O}_{4}(1.25 \mathrm{wt} \%)$ Aggregates (b) (e) HRTEM Images Illustrating the Different Shapes of Assembled M-Fe ${ }_{3} \mathrm{O}_{4}$ NPs on PS- $b$-P2VP Sphere Surface (f) HRTEM Image of (e) with Higher Magnification

When the content of $\mathrm{M}-\mathrm{Fe}_{3} \mathrm{O}_{4}$ was $2.5 \mathrm{wt} \%$, the mixture solution was still clear at the beginning, but some deposit appeared after equilibration, indicating the formation of large aggregates. Figure $5 \mathrm{a}$ and c-d are typical TEM images sampling clear solution and the deposit of PS- $b-\mathrm{P} 2 \mathrm{VP} / \mathrm{M}-\mathrm{Fe}_{3} \mathrm{O}_{4}$ (2.5 wt\%) mixture. The TEM image (Fig.5a) and its HRTEM image (Fig. 5b) show the biphasic structure with $\mathrm{M}-\mathrm{Fe}_{3} \mathrm{O}_{4} \mathrm{NPs}$ assembled on one side (the black part) and copolymers (the gray part) 
on the other. However, large copolymer microspheres and $\mathrm{M}-\mathrm{Fe}_{3} \mathrm{O}_{4} \mathrm{NPs}$ aggregates were observed for the deposit (Fig.5c and 5d). Peaks at about 300nm and 5.5 $\mathrm{mm}$ on the DLS curve (Fig.6b) were observed. The peak position at $300 \mathrm{~nm}$ is the same as that when the content of $\mathrm{M}-\mathrm{Fe}_{3} \mathrm{O}_{4}$ was 1.25 wt\%, which are consistent with the TEM observation (Fig. 5a). The peak at $5.5 \mu \mathrm{m}$ illustrated the formation of large PS- $b$-P2VP (Fig.5c) or PS- $b-\mathrm{P} 2 \mathrm{VP} / \mathrm{M}-\mathrm{Fe}_{3} \mathrm{O}_{4}$ aggregates (Fig. 5d). When the content of $\mathrm{M}-\mathrm{Fe}_{3} \mathrm{O}_{4}$ was increased further to $5.0 \mathrm{wt} \%$, precipitates were always observed. The DLS result (Fig.6c) was similar to that of Figure 6b. The difference between Fig.6b and c is the peak widths. Only the $\mathrm{BC}$ microspheres were observed in the supernatant fluid, indicating that $\mathrm{M}-\mathrm{Fe}_{3} \mathrm{O}_{4}$ NPs precipitated out completely when the content of $\mathrm{M}-\mathrm{Fe}_{3} \mathrm{O}_{4}$ was $5.0 \mathrm{wt} \%$.

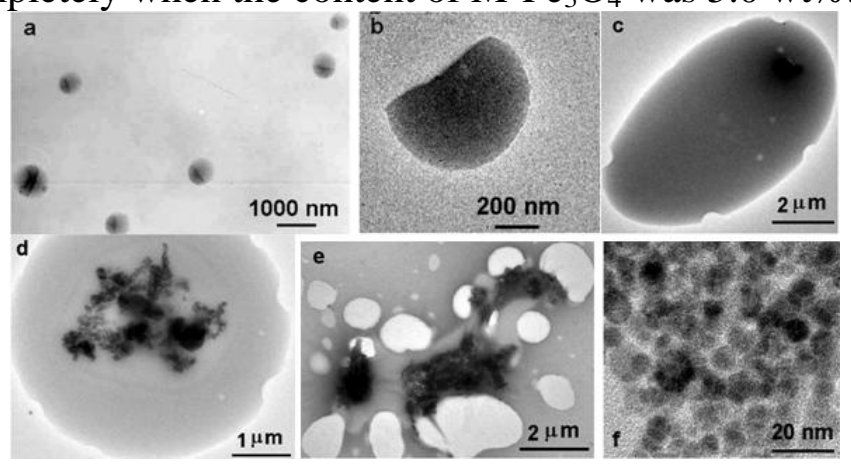

Fig.5 (a, b) TEM and HRTEM Images of the PS- $b$-P2VP/M-Fe ${ }_{3} \mathrm{O}_{4}(2.5 \mathrm{wt} \%)$ Aggregates, the Sample Taken from Clear Solution (c, d) TEM Images of the PS- $b$-P2VP/M-Fe ${ }_{3} \mathrm{O}_{4}(2.5 \mathrm{wt} \%)$

Aggregates, the Sample Taken from Deposit; (e, f) TEM and HRTEM Images of the $\mathrm{PS}-b$-P2VP/M-Fe ${ }_{3} \mathrm{O}_{4}(5.0 \mathrm{wt} \%)$ Aggregates

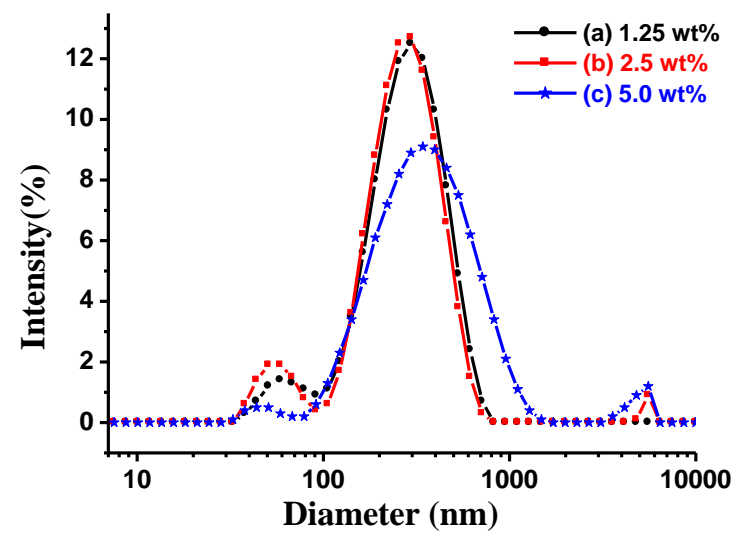

Fig.6 DLS Size Distributions of the PS- $b$-P2VP/M-Fe ${ }_{3} \mathrm{O}_{4}$ Aggregates in THF; the Contents of Magnetic $\mathrm{M}-\mathrm{Fe}_{3} \mathrm{O}_{4}$ NPs Relative to PS- $b$-P2VP are $1.25 \mathrm{wt} \%$ (Solid Circle), $2.5 \mathrm{wt} \%$ (Solid Square) and $5.0 \mathrm{wt} \%$ (Solid Star), Respectively

In this system, the organic shell of $\mathrm{M}-\mathrm{Fe}_{3} \mathrm{O}_{4} \mathrm{NPs}$ contains many mercapto groups. Hydrogen bonding interactions between the mercapto groups of the MUA ligands and the pyridine groups of the P2VP blocks drive the BCs to aggregate and $\mathrm{M}-\mathrm{Fe}_{3} \mathrm{O}_{4} \mathrm{NPs}$ tend to contact with the P2VP blocks to reduce the free energy of the system. This behavior causes the solubility of $\mathrm{P} 2 \mathrm{VP} / \mathrm{M}-\mathrm{Fe}_{3} \mathrm{O}_{4}$ to be significantly lower than that of the $\mathrm{P} 2 \mathrm{VP}$, and resulting in the formation of composite micelles or aggregates. The PS blocks and $\mathrm{P} 2 \mathrm{VP} / \mathrm{M}-\mathrm{Fe}_{3} \mathrm{O}_{4}$ blocks of the composite micelles or aggregates phase separate into a Janus-like structure, likely resulting from minimization of interfacial tensions. It was deduced from TEM images (Fig.3 and Fig.5a and 5b) that the $\mathrm{M}-\mathrm{Fe}_{3} \mathrm{O}_{4} \mathrm{NPs}$ might mainly distribute on the surface of PS- $b$-P2VP spheres due to the size of $\mathrm{M}-\mathrm{Fe}_{3} \mathrm{O}_{4}$ NPs are relatively large (about 10nm). Phase separation leads to the formation of the biphasic NPs observed in the 
microemulsion system. With the increase of the content of $\mathrm{M}-\mathrm{Fe}_{3} \mathrm{O}_{4}$ from $1.25 \mathrm{wt} \%$ to $2.5 \mathrm{wt} \%$, the number of $-\mathrm{SH}$ groups complexed with the pyridine units on the P2VP blocks increased. So the rigidity of the $\mathrm{P} 2 \mathrm{VP} / \mathrm{M}-\mathrm{Fe}_{3} \mathrm{O}_{4}$ blocks increases, which results in the smaller conformational entropy loss upon aggregating. Simultaneously, the solubility of the complexes in THF should decrease further. To minimize the interfacial area, large PS- $b-\mathrm{P} 2 \mathrm{VP} / \mathrm{M}-\mathrm{Fe}_{3} \mathrm{O}_{4}$ aggregates form. When the content of $\mathrm{M}-\mathrm{Fe}_{3} \mathrm{O}_{4}$ was $5.0 \mathrm{wt} \%$, the behavior in the beginning of the forming the mixture may be similar to that when the content of $\mathrm{M}-\mathrm{Fe}_{3} \mathrm{O}_{4}$ was $2.5 \mathrm{wt} \%$. Due to the increased content of magnetic NPs, more and more magnetic NPs covered the surface of the PS- $b$-P2VP spheres. When their concentration reached saturation value, they agglomerated, driven by van der Waals interactions, resulting in the precipitate of $\mathrm{M}-\mathrm{Fe}_{3} \mathrm{O}_{4}$ NPs from the solution (Fig.5e). Fig.5f illustrates that the aggregates were assembled $\mathrm{M}-\mathrm{Fe}_{3} \mathrm{O}_{4} \mathrm{NPs}$.

\section{Conclusions}

A simple approach to preparation of organic-inorganic asymmetric composite particles from the co-assembly of BCs and $\mathrm{Fe}_{3} \mathrm{O}_{4} \mathrm{NPs}$ in solution was presented. PS- $b$-P2VP is present as unimers in THF. If a small amount of MUA modified $\mathrm{Fe}_{3} \mathrm{O}_{4}$ NPs is added to the PS- $b-\mathrm{P} 2 \mathrm{VP}$ solution, organic-inorganic asymmetric particles composed of the copolymers and $\mathrm{M}-\mathrm{Fe}_{3} \mathrm{O}_{4} \mathrm{NPs}$ can form. This approach could also be used to synthesize other types of asymmetric BCs/inorganic NPs, which should be useful as biological and chemical sensors.

\section{References}

[1] J. He, M.T. Perez, P. Zhang, Y. Liu, T. Babu, J. Gong, Z. Nie, A general approach to synthesize asymmetric hybrid nanoparticles by interfacial reactions, J. Am. Chem. Soc., 134(2012) 3639-3642.

[2] Y.D. Yin, Y. Lu, Y.N. Xia, A self-assembly approach to the formation of asymmetric dimers from monodispersed spherical colloids, J. Am. Chem. Soc., 123(2001) 771-772.

[3] X. Li, H. Yang, L.M. Xu, X.N. Fu, H.W. Guo, X.K. Zhang, Janus micelle formation induced by protonation/deprotonation of poly-(2-vinylpyridine)-block-poly(ethylene oxide) diblock copolymers. Macromol, Chem. Phys., 211(2010) 297-302.

[4] X. Fu, L. Song, J. Liu, X. Li, X. Zhang, Y. Jia, One-step approach for the preparation of organic-inorganic Janus-like particles by alkalization of polystyrene-block-poly(2-vinylpyridine)/ $\mathrm{FeCl} 3$ complex micelles, Macromol. Chem. Phys., 213(2012) 1663-1668.

[5] Y. Wang, H. Xu, Y. Ma, F. Guo, F. Wang, D. Shi, Facile one-pot synthesis and morphological control of asymmetric superparamagnetic composite nanoparticles, Langmuir, 27(2011) 7207-7212.

[6] H. Yu, M. Chen, P.M. Rice, S.X. Wang, R.L. White, S. Sun, Dumbbell-like bifunctional Au-Fe3O4 nanoparticles, Nano Lett., 5(2005) 379-382.

[7] M.J. Hostetler, J.J. Stokes, R.W. Murray, Infrared spectroscopy of three-dimensional self-assembled monolayers: N-alkanethiolate monolayers on gold cluster compounds, Langmuir, 12(1996) 3604-3612.

[8] A. Tlili, A. Abdelghani, S. Hleli, M.A. Maaref, Electrical characterization of a thiol SAM on gold as a first step for the fabrication of an immunosensors based on a quartz crystal microbalance, Sensors, 4(2004) 105-114. 